\title{
An iterative observer approach to harmonic
}

\section{estimation for multiple measurements in}

\section{power distribution systems}

\author{
Awajiokiche Ujile*, Zhengtao Ding* and Haiyu Li*
}

${ }^{*}$ School of Electrical and Electronic Engineering, University of Manchester,

Manchester, M13 9PL, United Kingdom

awajiokiche.ujile@manchester.ac.uk, zhengtao.ding@manchester.ac.uk, haiyu.li@manchester.ac.uk

\begin{abstract}
In the past decade, there has been a significant increase in the use of power electronic components in the design of household and industrial equipment. The use of power electronic control based renewable energy resources, electric vehicles and other residential nonlinear loads may result in significant increases in injection levels of harmonics across a power system. Hence, it is important for utility companies to ascertain the exact harmonic levels present in terms of the amplitude and phase of each harmonic order. This paper provides a mathematical basis for distribution system state space equations to formulate an iterative observer, which can simultaneously estimate harmonics present in a number of measurements taken from the power system. The method not only improves the computation time and provides real-time data for harmonic monitoring, but also performs wide area harmonic estimation for harmonic observability. Simulations and comparisons are provided to illustrate the performances of the proposed method against those obtained using a Kalman filter and Fast Fourier Transform(FFT). A number of scenarios such as measurement noise and change in amplitude of harmonic injections are simulated to verify the accuracy of the proposed approach and the results are included.
\end{abstract}

Keywords - Harmonic analysis, observers, power distribution system, total harmonic distortion, harmonic estimation, power quality 


\section{INTRODUCTION}

The increasing use of power electronic devices in the design of household and industrial equipment has caused an increase in the level of harmonics in power distribution systems. Various organisations have created regulations to put restrictions on the allowable harmonic content in power distribution systems (IEEE, 1993). To enable utility companies comply with these regulations, it is imperative that they are aware of the level of harmonic injections to enable them design a suitable mitigating technique accordingly.

Harmonic estimation entails determining the characteristics of harmonic injections in a distorted signal. These characteristics include the amplitude and phase of each harmonic component present in a measured signal. The estimation and rejection of periodic disturbances has been extensively explored in literature. In (Wu et al., 2014), an adaptive regulation method for estimation of multiple sinusoidal exogenous disturbance inputs, where the disturbance is not available for measurement is presented. A Youla parameterization of stabilizing controllers is used to achieve regulation in the system. Observers have also been widely used in literature for sinusoidal disturbance estimation and compensation (Chen et al., 2014, Wen et al., 2011). However, their application to harmonic estimation in multiple output power distribution systems has not been explored.

Several methods have been proposed in literature for carrying out harmonic estimation. The Fast Fourier Transform (FFT) has been the most common method of harmonic estimation for decades (Chang et al., 2000, Harris, 1978). Transformation of the signals from the time domain to the frequency domain enables the FFT to accurately track the harmonic components. An advantage of the FFT is its ease of computation and accuracy. However, the presence of interharmonics or a deviation in the fundamental frequency of the system may result in inaccuracies such as aliasing, leakage and picket fence effects (Qian et al., 2007, Chang et al., 2008). In addition, the FFT can only perform estimation on one measurement at a time, hence, multiple point harmonic estimation cannot be achieved.

Other methods which have been proposed in literature for harmonic estimation include the Kalman filter as shown in Ma and Girgis (1996) and Kanieski et al. (2013), modifications of the Kalman filter such as the extended Kalman filter, unscented Kalman filter (Regulski and Terzija, 2012), adaptive Kalman filter (Reza et al., 2012) and ensemble Kalman filter (Ray 
and Subudhi, 2012). A comparative study of harmonic estimation methods is given in Chen and Chen (2014). Other non-Kalman filter methods for harmonic estimation proposed include estimation of signal parameters based on the rotational invariance technique (ESPRIT) (Jain and Singh, 2013, Jain et al., 2013) and artificial neural network techniques (Jain et al., 2011, Chang et al., 2009). These methods have been used to accurately estimate the amplitude and phase of harmonics in measured signals. However, they do not take into account the need for estimation of harmonics in multiple signals simultaneously. Estimation is carried out on a single measurement signal taken at a particular point in the power system. If, however, there is a need to obtain harmonic estimates from a separate point, utility companies need to carry out estimation again to obtain the required information. Although the possibility that the Kalman filter can be modified to perform simultaneous harmonic estimation on a number of measurements exists, the computation time involved is large and estimation is still carried out regardless of the distortion level of each measurement.

In this paper, a novel approach to simultaneous harmonic estimation in multiple measurements taken from different points of the power distribution system is presented. These measurements taken are synchronised in real time using phasor measurement units (PMU). The multiple point approach to harmonic estimation is important because it provides estimates of the amplitude and phase of harmonic injections in various measurements taken across a given power system. This information is useful because it provides information on the various harmonic injection levels throughout the power system as well as the harmonic flow. The iterative observer for harmonic estimation is derived from the utilisation of observers for estimation of periodic disturbances proposed in Ding (2008) and Friedland (1987). The assumption that a previous observer has been designed for the system without the consideration of the harmonic disturbance is the framework on which the iterative observer algorithm is built. Harmonic frequencies are modelled as disturbances to the system and the characteristics of these harmonics (amplitude and phase) are estimated from measurements. The approach presented in this paper is a continuation of the work done in Ujile and Ding (2014). The algorithm presented in Ujile and Ding (2014) can only be used for harmonic estimation on one measurement at a time. This paper provides a more general algorithm for harmonic estimation in both single output and multiple output power distribution systems. This algorithm is applied 
to all measurements simultaneously to determine the amplitude and phase of all frequencies present in the distorted signals. The developed algorithm automatically computes the total harmonic distortion (THD) of the system measurements and decides if harmonic estimation is required. If some of the measured signals are not distorted, harmonic estimation is not carried out on those signals. Harmonic estimation is only carried out if the percentage THD of the residual exceeds a certain threshold. The threshold for termination of iteration is set based on the regulatory requirement which states that for systems below $69 \mathrm{kV}$, the THD of each individual harmonic order should not exceed 3\% (IEEE, 1993). Therefore, if the THD of the residual with respect to the fundamental frequency exceeds the threshold, the iterative observer is employed for harmonic estimation. This threshold may be modified to suit the needs of the utility companies concerned.

The measurement points are chosen to ensure complete observability of the power system. As a result of the observability condition, the results obtained from harmonic estimation of all measurements may be used to map the flow of harmonics from their sources to other parts of the power system. This is an improvement on previous harmonic estimation methods as the results obtained can be utilised for other purposes such as harmonic source identification and filtering. Harmonics are generally propagated from the nonlinear loads towards the supply. Therefore, the determination of the harmonic amplitudes enables utility companies to track the harmonic flow from the source to supply. These amplitudes tend to reduce with respect to the proximity of the buses to the harmonic source. The FFT only carries out harmonic estimation on a single measurement signal at any given time, hence it is unable to provide information on the harmonic propagation in the power system.

Furthermore, the Kalman filter approach to harmonic estimation requires prior knowledge of the process and measurement noise covariances. This is not the case with the iterative observer as the observer gain may be designed using pole placement methods. An added advantage of the iterative observer algorithm is its application to smart energy meters. This may be instrumental in determining the exact harmonic contribution of each customer. 


\section{PRoblem FORMUlation}

Consider a power distribution system represented in a linearised state space form as:

$$
\begin{aligned}
& \dot{x}=A x+B u+E w \\
& y=C x
\end{aligned}
$$

where $x \in \mathbb{R}^{n}$ is the state vector, $y$ represents the measurements, $u \in \mathbb{R}$ is the system input, $A, B, C$ are matrices of appropriate dimensions with $C \in R^{m \times n}, m>1, E$ is the harmonic disturbance matrix which is unknown, $w$ is the harmonic disturbance, given by

$$
w(t)=\sum_{h=2}^{n} A_{h} \sin \left(\omega_{h} t+\phi_{h}\right)
$$

where for $h=2 \ldots n, A_{h}, \phi_{h} \in \mathbb{R}, \omega_{h}=2 \pi f h, f$ is the fundamental frequency and $h$ is the harmonic order.

The dynamic model of $w_{h}$ is represented as the linear exosystem:

$$
\begin{gathered}
\dot{w}_{h}=S_{h} w_{h} \\
\mu_{h}=g^{T} w_{h}
\end{gathered}
$$

where $S_{h}=\left[\begin{array}{cc}0 & \omega_{h} \\ -\omega_{h} & 0\end{array}\right], g^{T}=\left[\begin{array}{ll}1 & 0\end{array}\right]$ and $w_{h} \in \mathbb{R}^{2}$ is the harmonic state.

Remark 1. The nonlinear loads in power systems cause harmonic injections by altering the current waveform irrespective of the sinusoidal nature of the input voltage. These harmonic injections are periodic non-sinusoidal waveforms which alter the sinusoidal input voltage. Taking the Fourier transform of the measured signal yields the individual harmonics where each harmonic order consists of two states as shown in (4).

$$
\left[\begin{array}{l}
w_{1, h} \\
w_{2, h}
\end{array}\right]=\left[\begin{array}{c}
A_{h} \sin \left(\omega_{h} t+\phi_{h}\right) \\
A_{h} \cos \left(\omega_{h} t+\phi_{h}\right)
\end{array}\right]
$$

In addition, $h=1$, which is the fundamental frequency, is not considered as a harmonic disturbance to the power system. The system operates at this base frequency and every other frequency contained in the measurements is considered to be a harmonic disturbance.

Assumption 1. The harmonic disturbance is not directly measured. Hence, the state space 
model in (1) has no indication of the harmonic content in the output.

Assumption 2. The pair $\left(g^{T}, S_{h}\right)$ is observable.

Remark 2. The state vector, $x$, consists of bus voltages and branch currents in the power distribution system.

The aim of the work done in this paper is to design an iterative observer to simultaneously carry out harmonic estimation in multiple power system measurements. This includes the determination of the amplitude, $A_{i}$, and phase, $\phi_{i}$, of each harmonic order present in the measurements. The harmonics are modelled as a disturbance given in (1). The power system is modelled as a process at the fundamental frequency and the individual harmonic components are taken as disturbances as shown in Fig. 1.

\section{ITERATIVE OBSERVER DESIGN FOR HARMONIC ESTIMATION IN SINGLE OUTPUT}

\section{MEASUREMENTS}

An iterative observer is proposed in this section for the estimation of harmonics in a voltage or current measurement. Suppose the $i^{\text {th }}$ harmonic order is to be estimated, with dynamics given as

$$
\dot{w}_{i}=S_{i} w_{i}
$$

The system dynamics and exogenous disturbance dynamics are augmented to yield

$$
\left[\begin{array}{c}
\dot{x} \\
\dot{w}_{i}
\end{array}\right]=\left[\begin{array}{cc}
A & 0 \\
0 & S_{i}
\end{array}\right]\left[\begin{array}{l}
x \\
w_{i}
\end{array}\right]+\left[\begin{array}{l}
B \\
0
\end{array}\right] u
$$

Applying the Luenberger observer (Luenberger, 1966) to the augmented system in (6) gives

$$
\left[\begin{array}{c}
\dot{\hat{x}} \\
\dot{\hat{w}}_{i}
\end{array}\right]=\left[\begin{array}{cc}
A & 0 \\
0 & S_{i}
\end{array}\right]\left[\begin{array}{l}
\hat{x} \\
\hat{w}_{i}
\end{array}\right]+\left[\begin{array}{l}
B \\
0
\end{array}\right] u+\left[\begin{array}{c}
L \\
L_{i}
\end{array}\right](y-C \hat{x})
$$

Separating this augmented observer into equations for the process state estimate and the exogenous harmonic disturbance estimate, we have

$$
\begin{aligned}
& \dot{\hat{x}}=A \hat{x}+B u+L\left(y-C \hat{x}-g^{T} \hat{w}_{i}\right) \\
& \dot{\hat{w}}_{i}=S_{i} \hat{w}_{i}+L_{i}\left(y-C \hat{x}-g^{T} \hat{w}_{i}\right)
\end{aligned}
$$




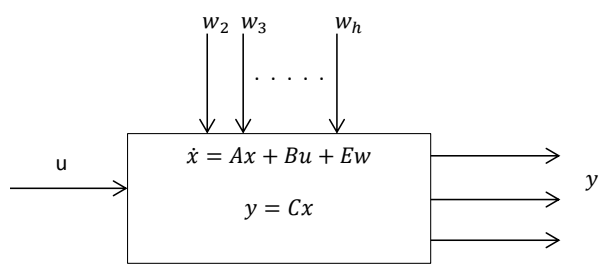

Fig. 1. Power distribution system state space representation

The state estimate $\hat{x}$ is designed based on an inclusion of the system states, $x$ and the disturbance state, $w$. Suppose an observer has been designed previously for power system state estimation without the consideration of the harmonic disturbance, this observer is given as

$$
\dot{\tilde{x}}=A \tilde{x}+B u+\tilde{L}(y-C \tilde{x})
$$

where $\tilde{L}$ is designed such that $(A-\tilde{L} C)$ is Hurwitz. The observer gain may be designed based on pole placement methods as depicted in Selvajyothi and Janakiraman (2008). The residual $y-C \tilde{x}$, is orthogonal to the harmonic states to be estimated, $w$.

The state variable is expressed as a summation of the actual system states and the exogenous states, given by

$$
\hat{x}=\tilde{x}+V_{i} \hat{w}_{i}
$$

where $V_{i}$ is a weighting matrix. Taking derivatives of (10) with respect to time, we have

$$
\begin{aligned}
\dot{\hat{x}} & =A \tilde{x}+B u+\left(\tilde{L}+V_{i} L_{i}\right)(y-C \tilde{x})+\left(V_{i} S_{i}-V_{i} L_{i} C V_{i}-V_{i} L_{i} g^{T}\right) \hat{w}_{i} \\
& =A \tilde{x}+B u+\left(\tilde{L}+V_{i} L_{i}\right)(y-C \tilde{x})+V_{i}\left(S_{i}-L_{i}\left(C V_{i}+g^{T}\right)\right)
\end{aligned}
$$

However, from (8):

$$
\dot{\hat{x}}=A \hat{x}+B u+L\left(y-C \hat{x}-g^{T} \hat{w}_{i}\right)
$$

Hence

$$
\dot{\hat{x}}=A \tilde{x}+B u+L(y-C \tilde{x})+\left(A V_{i}-L C V_{i}-L g^{T}\right) \hat{w}_{i}
$$

Comparing (11) and (13):

$$
L=\tilde{L}+V_{i} L_{i}
$$




$$
\begin{aligned}
V_{i} S_{i}-V_{i} L_{i} C V_{i}-V_{i} L_{i} g^{T} & =A V_{i}-L C V_{i}-L g^{T} \\
V_{i} S_{i}-(A-\tilde{L} C) V_{i} & =-\tilde{L} g^{T}
\end{aligned}
$$

(15) represents a Sylvester equation of the form $G e+e H=Q$, with $e$ calculated as follows:

$$
\begin{array}{r}
(I \otimes G+H \otimes I) \operatorname{vec}(e)=\operatorname{vec}(Q) \\
\operatorname{vec}(e)=(I \otimes G+H \otimes I)^{-1} \operatorname{vec}(Q)
\end{array}
$$

$V_{i}$ is calculated using the same method in (16).

It is important to note that (15) is only applicable when a single harmonic order is to be estimated. Hence the estimate for the $i^{\text {th }}$ harmonic order is shown in (17).

$$
\dot{\hat{w}}_{i}=S_{i} \hat{w}_{i}+L_{i}\left(y-C \tilde{x}-\left(C V_{i}+g^{T}\right) \hat{w}_{i}\right)
$$

Suppose an additional harmonic order is to be estimated. We define a set of positive integers $\mathcal{H}=\left\{h_{i}\right\}$ for $i=1 \ldots n$. To carry out estimation of the initial harmonic, where $i=1$, (17) is used to carry out estimation. For $h_{i+1}$, we have

$$
\dot{\hat{w}}_{h, i+1}=S_{h, i+1} \hat{w}_{h, i+1}+L_{h, i+1}\left(y-C \hat{x}-g^{T} \hat{w}_{h, i+1}\right)
$$

As a result of the additional harmonic order to be estimated, the system state estimate becomes

$$
\hat{x}=\tilde{x}+\sum_{i=1}^{n} V_{h, i} \hat{w}_{h, i}
$$

Taking derivatives of (19) with time

$$
\begin{aligned}
\dot{\hat{x}}= & \dot{\tilde{x}}+\sum_{i=1}^{n} V_{h, i} \dot{\hat{w}}_{h, i} \\
= & A \tilde{x}+B u+\left(\tilde{L}+\sum_{i=1}^{n} V_{h, i} L_{h, i}\right)(y-C \tilde{x})+\sum_{i=1}^{n} V_{h, i}\left(S_{h, i}-L_{h, i}\left(C V_{h, i}\right.\right. \\
& \left.\left.+g^{T}\right)\right) \hat{w}_{h, i}
\end{aligned}
$$

However, from (8), we have

$$
\dot{\hat{x}}=A \tilde{x}+B u+L(y-C \tilde{x})+\sum_{i=1}^{n}\left((A-L C) V_{h, i}-L g^{T}\right) \hat{w}_{h, i}
$$


Comparing (21) and (22)

$$
L=\tilde{L}+\sum_{i=1}^{n} V_{h, i} L_{h, i}
$$

Hence, for $i=1$,

$$
\begin{gathered}
V_{h, 1} S_{h, 1}-(A-\tilde{L} C) V_{h, 1}=-\tilde{L} g^{T} \\
\dot{\hat{w}}_{h, 1}=S_{h, 1} \hat{w}_{1}+L_{h, 1}\left(y-C \tilde{x}-\left(C V_{h, 1}+g^{T}\right) \hat{w}_{h, 1}\right)
\end{gathered}
$$

and for $i=2 \ldots n$

$$
V_{h, i} S_{h, i}-\left(A-\left(\tilde{L}+\sum_{j=1}^{n-1} V_{j} L_{j}\right) C\right) V_{h, i}=-\left(\tilde{L}+\sum_{j=1}^{n-1} V_{j} L_{j}\right) g^{T}
$$

Therefore the harmonic estimate is given by

$$
\dot{\hat{w}}_{h, i}=S_{h, i} \hat{w}_{h, i}+L_{h, i}\left(y-C \tilde{x}-\sum_{i=2}^{n}\left(C V_{h, i}+g^{T}\right) \hat{w}_{h, i}\right)
$$

where $L_{h, i}$ for $i=1 \ldots n$ is designed such that $S_{h, i}-L_{h, i} g^{T}$ is Hurwitz.

Theorem 1. For a given power distribution system with dynamic equation (1), containing harmonics which are represented as a linear exosystem given in (3), estimation of the harmonic content can be produced by (17) for $\mathcal{H}_{i}=\left\{h_{2} \ldots h_{n}\right\}$. Additional harmonics $\mathcal{H}_{n+1}$, can be estimated by using the iterative observer of the form (28).

$$
\dot{\hat{w}}_{h, n+1}=S_{h, n+1} \hat{w}_{h, n+1}+L_{h, n+1}\left(y-C \tilde{x}-\sum_{i=2}^{n}\left(C V_{h, i}+g^{T}\right) \hat{w}_{h, n+1}\right)
$$

Proof. The residual, $y-C \tilde{x}-\sum_{i=2}^{n}\left(C V_{h, i}+g^{T}\right) \hat{w}_{h, n+1}$ approaches zero asymptotically when all harmonic frequencies have been estimated. Consider the time derivatives of the estimation error

$$
\dot{e}=\dot{x}-\dot{\hat{x}}
$$

Substituting (19) and (28) we have

$$
\begin{aligned}
\dot{e} & =A x+B u-A \tilde{x}-A \sum_{i=2}^{n} V_{h, i} \hat{w}_{h, n+1}-B u-L\left(y-C \tilde{x}-C \sum_{i=2}^{n} V_{h, i} \hat{w}_{h, n+1}\right. \\
& =(A-L C)\left(x-\tilde{x}-\sum_{i=2}^{n} V_{h, i} \hat{w}_{h, n+1}\right)
\end{aligned}
$$


Equation (30) shows that the estimation error approaches zero asymptotically when the state variables are expressed as shown in (19).

For $i=n+1$, the estimation error for harmonic estimates is given by $e_{n+1}=w_{h, n+1}-$ $\hat{w}_{h, n+1}$. Taking the time derivative of this error yields

$$
\dot{e}_{h, n+1}=\left(S_{h, n+1}-L_{h, n+1} g^{T}\right) e_{h, n+1}-L_{h, n+1}\left(y-g^{T} \sum_{k=2}^{n} \hat{w}_{h, k}\right)
$$

for $k \neq h$, where $\left(S_{h, n+1}-L_{h, n+1} g^{T}\right)$ is Hurwitz and $\left(y-g^{T} \sum_{k=2}^{n} \hat{w}_{h, k}\right)$ is orthogonal to the harmonic frequency, $h$. As a result of the asymptotic convergence of $y-g^{T} \sum_{k=2}^{n} \hat{w}_{h, k}$ to zero for $i=2 \ldots n$, this result holds true for $i=n+1$. This completes the proof.

\section{SimUltanEOUS HARMONIC ESTIMATION IN POWER DISTRIBUTION SYSTEMS}

A multiple point approach to the estimation of harmonics in power distribution systems is presented. The advantage of this method is its ability to provide an approximation of the harmonic propagation in a given power system. The iterative observer is applied to a number of measurements to simultaneously obtain the amplitudes and phase angles of all the harmonic components present in these signals. The location of the measurement points are chosen to ensure complete observability (Gou and Abur, 2001, Shafiee Rad et al., 2013, Castillo et al., 2006). If the measurement points satisfy the observability criteria, the harmonic estimation approach presented may be used to determine the harmonic levels at all system buses. This information is relevant to utility companies to enable them determine the source of harmonic injections in the power system and apply a mitigating technique accordingly. The harmonic levels at a certain bus is the approximate summation of all harmonic injections from adjacent buses (Abu-Hashim et al., 1999). Hence, the multiple point approach can be utilised to map the flow of harmonics from its sources to other parts of the system.

Application of the multiple point approach requires modification of certain parameters in the iterative observer algorithm. Evidently, the $A, B, C$ matrices which represent the state space dynamics remain unchanged. However, the observer gain, $L_{h, i}$, is modified to accommodate the number of measurements taken and the number of harmonic orders to be 
estimated. For $i=1 \ldots n$,

$$
S_{h, i}=\left[\begin{array}{cccc}
S_{h, i}^{1} & 0 & \ldots & 0 \\
0 & S_{h, i}^{2} & \ldots & 0 \\
\vdots & \vdots & \ddots & \vdots \\
0 & 0 & \ldots & S_{h, i}^{m}
\end{array}\right]
$$

where $m$ represents the number of measurements and $S_{h, i} \in \mathbb{R}^{2 m \times 2 m}$. The observer gain, $L_{h, i} \in \mathbb{R}^{2 m \times m}$ is designed such that $S_{h, i}-L_{h, i} C_{h, i}$ is Hurwitz. $g^{T} \in \mathbb{R}^{m \times 2 m}$ is given by

$$
g^{T}=\left[\begin{array}{ccccc}
1 & 0 & 0 & \ldots & 0 \\
0 & 1 & 0 & \ldots & 0 \\
\vdots & \vdots & \ddots & \vdots & \\
0 & 0 & 0 & 1 & 0
\end{array}\right]
$$

The THD of the residuals for all the measurements are checked to ensure that they comply with the set threshold for termination of iteration. Measurements which fail to meet the criteria are not estimated and as a result, the parameters, $S_{h, i}, g^{T}$ and $L_{h, i}$ are adjusted accordingly.

For a general case of multiple point harmonic estimation in a given power distribution system, the algorithm for harmonic estimation in the measured voltage or current signal using the iterative observer is given in algorithm 1. From the algorithm, the THD of the residual of the initial estimate, $\tilde{x}$, is checked to ensure that there is a need for carrying out harmonic estimation. $T$ is the threshold for termination of iteration and this value may be varied depending on the needs of the utility companies concerned.

\section{CASE STUDY}

A one-line diagram of a distribution feeder is shown in Fig. 2, with specifications taken from Abu-Hashim et al. (1999). The fundamental frequency is taken as $50 \mathrm{~Hz}$ and linear loads are connected at buses 34, 45, 46, 71, 75, 92, 52 and 911. These linear loads are modelled as constant RL impedances and lines are represented by their $\pi$ models (AbuHashim et al., 1999). The $A, B, C$ matrices are determined using the approach explained in Mamis (2003) and the appendix of Cisneros-Magaña and Medina (2012). Measurement points are chosen based on sequential elimination and binary integer linear programming as shown 

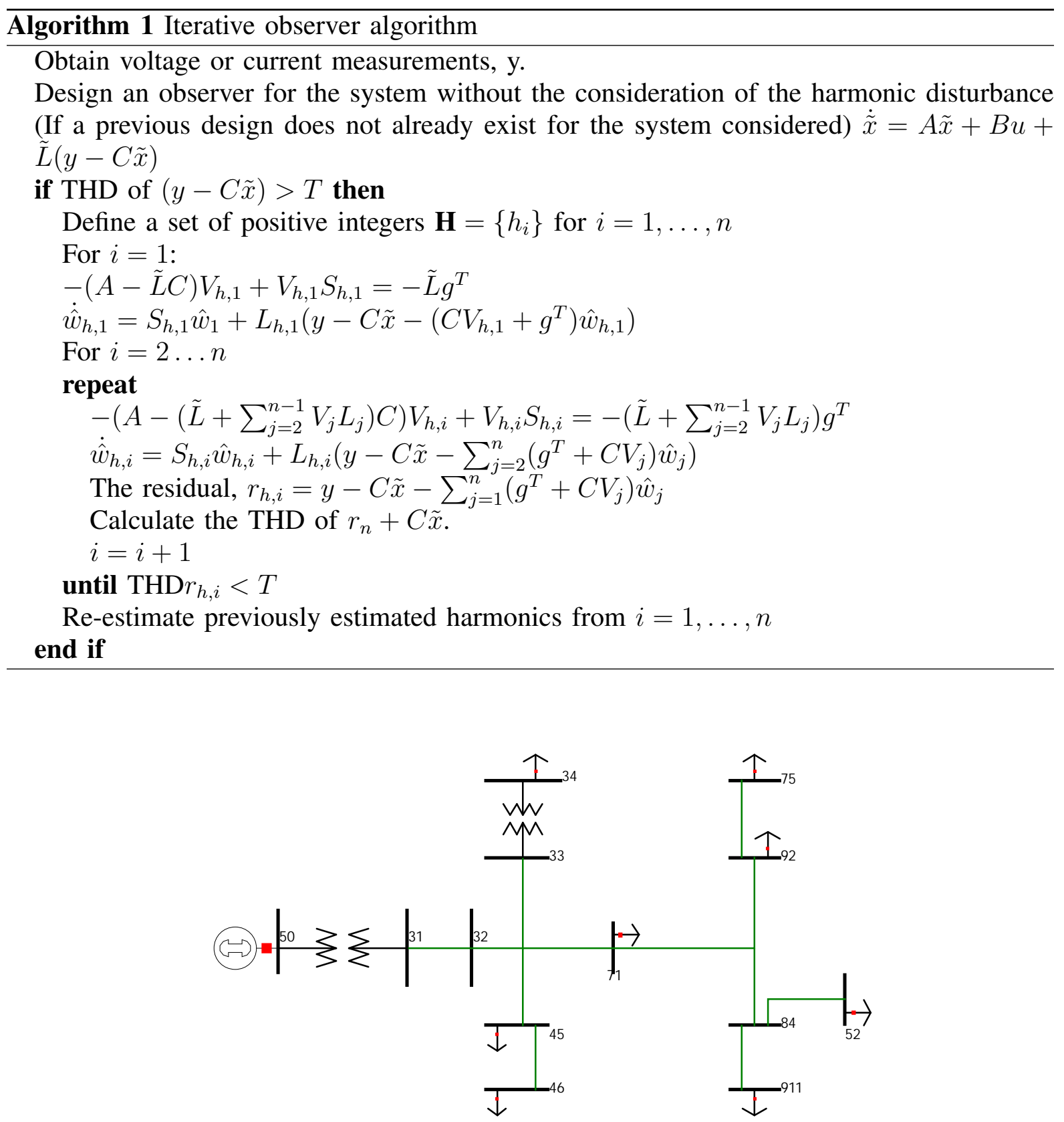

Fig. 2. One-line diagram of the IEEE 13-bus feeder (PowerWorld, 2012)

in Sodhi et al. (2010) to ensure complete observability. The observability criteria may be useful in further studies such as harmonic state estimation and harmonic source identification (Arrillaga et al., 2001, Shafiee Rad et al., 2013). Twelve measurements are taken from the feeder of which six are voltage measurements and the other six are current measurements. These measurements include $V_{31}, V_{33}, V_{45}, V_{92}, V_{71}, V_{84}, I_{31-32}, I_{33-34}, I_{45-46}, I_{32-71}, I_{92-75}$ and $I_{84-911}$ where $V_{i}$ represents voltage measurements at bus $i$ and $I_{i-j}$ represents current measurement at branch $i-j$. The nonlinear loads are modelled as linear loads in parallel 
with current sources, and, for the purpose of harmonic studies, two harmonic sources are assumed to be present in the feeder, at buses 46 and 75, with spectra shown in Tables I and II respectively. The iterative observer is applied to all measurements simultaneously, to determine the harmonic propagation throughout the system. The threshold for harmonic estimation is set at $0.5 \%$ which is below the maximum allowable THD in power distribution systems below $69 \mathrm{kV}$ as specified in (IEEE, 1993). Measurements with THD greater than this are subjected to the iterative observer algorithm to ascertain the harmonic levels in the distribution feeder. The measurements taken at a specific bus do not exactly portray the harmonic content injected at that bus. They represent a combination of the harmonic content at the buses adjacent to the buses where the measurements are taken.

A number of scenarios are simulated to verify the accuracy and robustness of the proposed method. The results obtained from the iterative observer approach are compared to those obtained using the Kalman filter and FFT.

\section{A. Normal operating condition}

The THD of all measurements is analysed to ensure compliance with the set threshold. The THD of all measurements is shown in Table III. Measurements which have the THD above the threshold are $V_{92}, V_{71}, V_{84}, V_{45}, I_{45-46}$ and $I_{92-75}$. Therefore harmonic estimation is only carried out for these measurements since they are the distorted signals. The iterative observer algorithm is applied to the distorted measurements and Table IV shows the THD after each harmonic order is estimated until all harmonic amplitudes and phase angles have been accurately estimated, where $h$ is the harmonic order. From the table, it is apparent that the THD after estimation of the fifth harmonic only exceeds the threshold for the measurements $V_{92}$ and $I_{92-75}$. The THD after estimation of other measurements is below the threshold so iteration is terminated. From the harmonic spectra for both harmonic sources at buses 46 and 75 , it is evident that the seventh harmonic only exists at bus 75 and bus 92 is the closest measurement point to bus 75 . Therefore the accuracy of the harmonic estimates is established.

Table V summarises a comparison between the FFT, Kalman filter (KF) and iterative observer (IO) methods of harmonic estimation for measurements with THD above the threshold, where $M$ represents the amplitude expressed as a percentage of the fundamental frequency and $P$ is the phase angle in degrees. For ease of comparison, the Kalman gain is equivalent 
TABLE I

HARMONIC SPECTRUM AT BUS 46

\begin{tabular}{c|c|c}
\hline Harmonic order & Amplitude $(\%)$ & Phase $\left(^{\circ}\right)$ \\
\hline Fundamental $(50 \mathrm{~Hz})$ & 100.00 & 95.1 \\
3rd $(150 \mathrm{~Hz})$ & 14.98 & 178.3 \\
5th $(250 \mathrm{~Hz})$ & 5.22 & 243.9 \\
\hline
\end{tabular}

TABLE II

HARMONIC SPECTRUM AT BUS 75

\begin{tabular}{c|c|c}
\hline Harmonic order & Amplitude $(\%)$ & $\operatorname{Phase}\left(^{\circ}\right)$ \\
\hline Fundamental $(50 \mathrm{~Hz})$ & 100.00 & -56.7 \\
3rd $(150 \mathrm{~Hz})$ & 13.96 & 216.7 \\
5th $(250 \mathrm{~Hz})$ & 4.68 & 282.3 \\
7th $(350 \mathrm{~Hz})$ & 1.70 & 81.0 \\
\hline
\end{tabular}

to the observer gain. The THD of the residual after estimation of the third harmonic for measurements $V_{92}, V_{71}, V_{84}$ and $V_{45}$ are $0.397,0.251,0.250$ and 0.264 respectively as shown in Table IV. As a result, the iterative observer terminates iteration for these measurements after estimation of the third harmonic. On the contrary, the residuals of $I_{45-46}$ and $I_{92-75}$ are above the threshold, therefore iteration continues. From table V, harmonic estimation is only carried out extensively for the $I_{92-75}$ and $I_{45-46}$ measurements. In addition, the flow of harmonics may be inferred from the results shown in Table III. These values show that the harmonic distortion in both voltage and current measurements diminishes as the harmonic currents flow from the harmonic source to the system source. The waveforms in Fig. 3 and 4 show a comparison between the iterative observer estimates and the Kalman filter estimates for the $I_{45-46}$ and $I_{92-75}$ measurements. These measurements are used to depict this comparison as they have the most harmonic distortion due to their proximity to the harmonic sources at bus 46 and 75 . The figure shows that the estimates obtained from both methods are approximately equivalent, using the same estimation gain for the Kalman filter and iterative observer. The results from table $\mathrm{V}$ also show that the iterative observer yields approximately similar results to the FFT and Kalman filter methods. However, the iterative observer is advantageous due to its added capability to iteratively estimate the harmonics based on their existence in the measurement. If the total harmonic distortion is below the set 
TABLE III

THD OF ALL MEASUREMENTS

\begin{tabular}{c|c|c|c|c|c|c|c|c|c|c|c|c}
\hline $\mathrm{y}$ & $V_{31}$ & $V_{33}$ & $V_{92}$ & $V_{71}$ & $V_{84}$ & $V_{45}$ & $I_{31-32}$ & $I_{33-34}$ & $I_{45-46}$ & $I_{32-71}$ & $I_{92-75}$ & $I_{84-911}$ \\
\hline THD & 0.00 & 0.38 & 0.82 & 0.52 & 0.52 & 0.55 & 0.18 & 0.23 & 15.53 & 0.11 & 14.26 & 0.31 \\
\hline
\end{tabular}

TABLE IV

THD OF ALL DISTORTED MEASUREMENTS AFTER ESTIMATION OF EACH HARMONIC FREQUENCY

\begin{tabular}{c|c|c|c|c|c|c}
\hline $\mathrm{h}$ & \multicolumn{6}{|c}{$\operatorname{THD}(\%)$} \\
\cline { 2 - 7 } & $V_{92}$ & $V_{71}$ & $V_{84}$ & $V_{45}$ & $I_{45-46}$ & $I_{92-75}$ \\
\hline 3 & 0.397 & 0.251 & 0.250 & 0.264 & 4.893 & 4.595 \\
\hline 5 & - & - & - & - & 0.044 & 1.506 \\
\hline 7 & - & - & - & - & 0.015 & 0.014 \\
\hline
\end{tabular}

threshold, then iteration is terminated thereby reducing computation time and cost.

\section{B. Harmonic estimation with changes in the amplitude of harmonics with time}

In actual power distribution systems, the amplitude of the harmonic injections may vary with time. Customers constantly use loads as and when needed and as a result, the harmonic injection levels may fluctuate with time. Suppose the current at bus 75 is given as:

$$
I_{75}=I_{1} \sin \left(\omega t+\phi_{1}\right)+I_{3} \sin \left(\omega t+\phi_{3}\right)+I_{5} \sin \left(\omega t+\phi_{5}\right)+I_{7} \sin \left(\omega t+\phi_{7}\right)
$$

where $\omega=2 \pi f, f=50 \mathrm{~Hz}$ and the time varying amplitudes are given as:

$$
\begin{gathered}
I_{3}= \begin{cases}0 & t<0.2 s \\
12.6 \% & 0.2 s<t<0.4 s \\
18.5 \% & t>0.4 s\end{cases} \\
I_{5}= \begin{cases}0 & t<0.2 s \\
4.14 \% & 0.2 s<t<0.4 s \\
0 & t>0.4 s\end{cases}
\end{gathered}
$$


TABLE V

COMPARISON OF METHODS FOR HARMONIC ESTIMATION FOR NORMAL OPERATING CONDITION

\begin{tabular}{|c|c|c|c|c|c|c|c|}
\hline \multirow{2}{*}{$y$} & \multirow{2}{*}{$h$} & \multicolumn{2}{|c|}{ FFT } & \multicolumn{2}{|c|}{$\mathrm{KF}$} & \multicolumn{2}{|c|}{ IO } \\
\hline & & $M(\%)$ & $P\left(^{\circ}\right)$ & $M(\%)$ & $P\left(^{\circ}\right)$ & $M(\%)$ & $P\left(^{\circ}\right)$ \\
\hline \multirow{3}{*}{$V_{92}$} & 3 & 0.75 & -68.9 & 0.71 & -81.8 & 0.72 & -81.0 \\
\hline & 5 & 0.48 & -9.5 & 0.39 & -12.4 & - & - \\
\hline & 7 & 0.07 & 137.1 & 0.12 & 162.2 & - & - \\
\hline \multirow{3}{*}{$V_{71}$} & 3 & 0.49 & -66.5 & 0.45 & -86.7 & 0.0 .46 & -86.0 \\
\hline & 5 & 0.34 & -13.0 & 0.26 & -18.9 & - & - \\
\hline & 7 & 0.03 & 49.7 & 0.05 & 164.9 & - & - \\
\hline \multirow{3}{*}{$V_{84}$} & 3 & 0.48 & -66.7 & 0.45 & -86.9 & 0.46 & -86.2 \\
\hline & 5 & 0.34 & -13.1 & 0.26 & -19.1 & - & - \\
\hline & 7 & 0.03 & 49.2 & 0.05 & 164.8 & - & - \\
\hline \multirow{3}{*}{$V_{45}$} & 3 & 0.47 & -79.6 & 0.48 & 260.1 & 0.49 & 260.9 \\
\hline & 5 & 0.35 & -22.9 & 0.28 & -31.5 & - & - \\
\hline & 7 & 0.06 & 9.2 & 0.01 & 164.5 & - & - \\
\hline \multirow{3}{*}{$I_{45-46}$} & 3 & 14.80 & -3.5 & 14.66 & -3.4 & 14.78 & -2.6 \\
\hline & 5 & 5.15 & 60.1 & 5.09 & 61.5 & 5.20 & 62.5 \\
\hline & 7 & 0.11 & 16.0 & 0.00 & 93.2 & - & - \\
\hline \multirow{3}{*}{$I_{92-75}$} & 3 & 13.53 & 34.4 & 13.44 & 34.9 & 13.59 & 35.7 \\
\hline & 5 & 4.47 & 99.0 & 4.47 & 100.8 & 4.75 & 101.7 \\
\hline & 7 & 1.59 & 260.3 & 1.66 & 258.1 & 1.84 & 257.7 \\
\hline
\end{tabular}
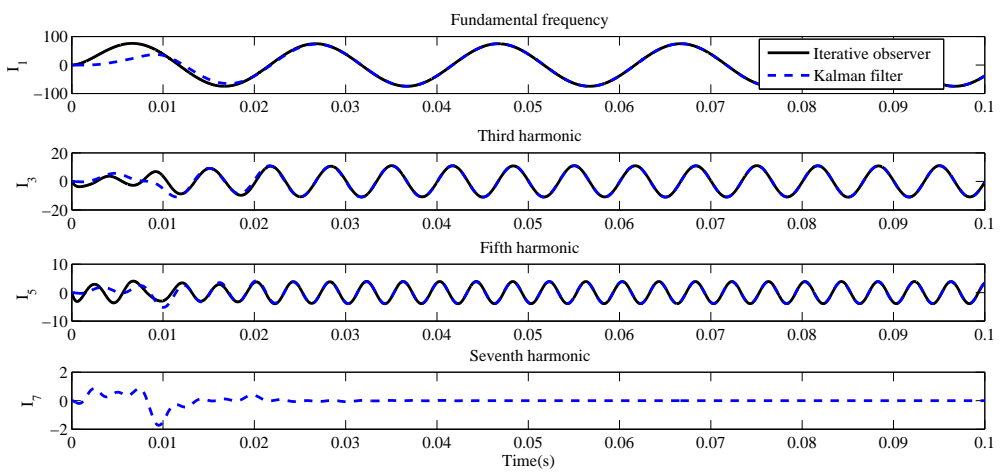

Fig. 3. Comparison between iterative observer and Kalman filter estimates for $I_{45-46}$ measurement

$$
I_{7}= \begin{cases}0 & t<0.2 s \\ 1.5 \% & 0.2 s<t<0.4 s \\ 0 & t>0.4 s\end{cases}
$$



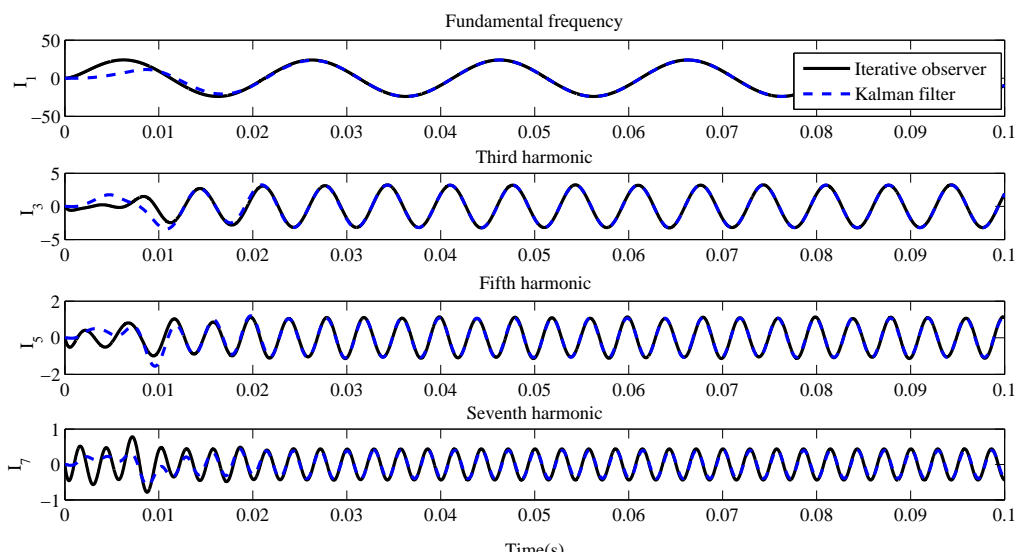

Fig. 4. Comparison between iterative observer and Kalman filter estimates for $I_{92-75}$ measurement

The iterative observer algorithm is applied to the measurements and the results are as shown in Fig 5. From the figure, it is apparent that at $t>0.2 s$, the third, fifth and seventh harmonics are injected into the feeder. For the third harmonic at $t<0.2 s$, there is no harmonic disturbance at bus 75 , hence the current $I_{92-75}$, which is the current measurement closest to that bus shows zero harmonic estimates for this interval. However, at the interval $0.2 s<t<0.4 s$, the third, fifth and seventh harmonics are estimated using the iterative observer. At $t>0.4 \mathrm{~s}$, only the third harmonic disturbance is estimated and this is reflected in the amplitude of the estimates at that time interval. In addition, the third harmonic amplitude increases and this is shown in Fig. 5. The figure shows that the iterative observer is capable of accurately estimating the amplitude of the harmonic injections in the measurements irrespective of the time instant in which the harmonic is injected. Also, the algorithm adjusts appropriately to sudden variations in the harmonic injections.

\section{Decaying DC component}

Consider a scenario where the current measurement at line 45-46 contains a slowly decaying DC component given by

$$
I(t)=I_{45-46}+I_{D C} e^{-\alpha t}
$$

where $I_{D C}=26.84 A$ and $\alpha=10$. All other system measurements do not contain the decaying DC component. Applying the iterative observer algorithm yields the results shown in Fig. 6. Figure 6a shows the harmonic amplitudes in the presence of the decaying DC component in 

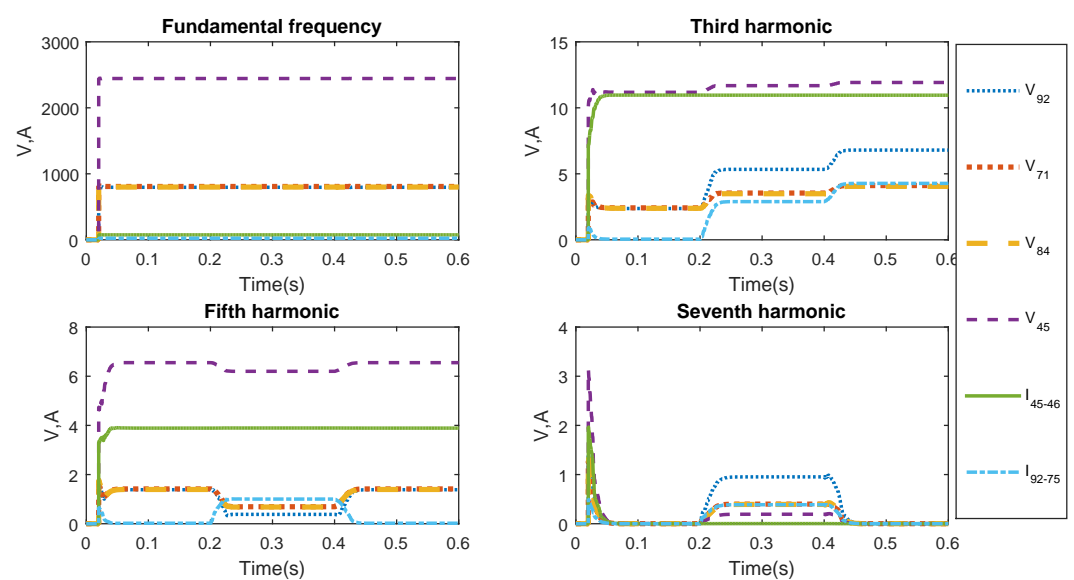

Fig. 5. Harmonic estimation with sudden change in the amplitude of harmonics with time
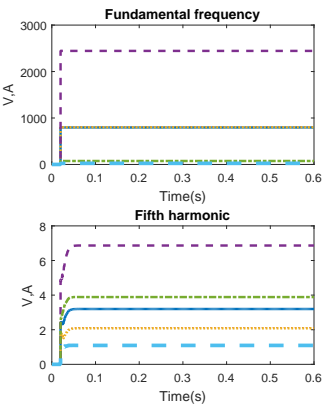

(a) Estimated amplitudes

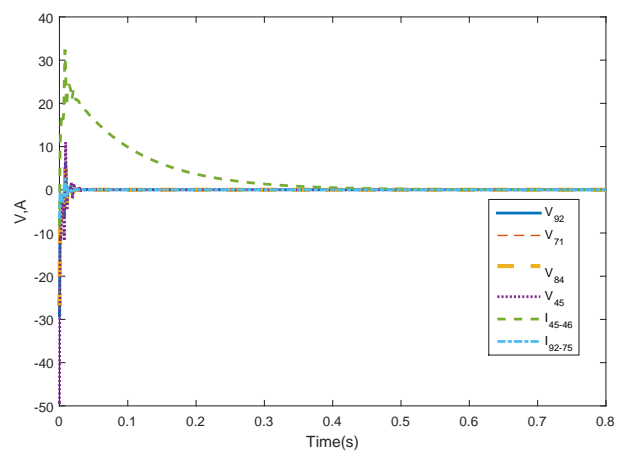

(b) Estimation error

Fig. 6. Harmonic estimates in the presence of decaying DC components in $I_{45-46}$

$I_{45-46}$. As seen in the figure, all harmonic estimates for the measurement affected, $I_{45-46}$ are accurate and the decay is seen in the estimation error in Fig. 6b. This verifies the robustness of the iterative observer algorithm to decaying DC components in measurements. The harmonic amplitudes and phase angles are accurately estimated irrespective of the presence of DC components in the measurements.

\section{Noisy measurements}

Consider a Gaussian distributed noise with variance of $5 \%$ and zero mean added to the measurements. The presence of noise in the measurements affects the THD. Table VI shows the THD of the noisy system measurements. The table shows that the presence of noise in the measurements provides an increase in the THD hence there are 8 measurements with THD exceeding the threshold. Figure 7 shows the residuals after harmonic estimation with noisy measurements. Despite the presence of noise in the measurements, all the estimation 
TABLE VI

THD OF SYSTEM MEASUREMENTS WITH NOISE

\begin{tabular}{c|c|c|c|c|c|c|c|c|c|c|c|c}
\hline $\mathrm{y}$ & $V_{31}$ & $V_{33}$ & $V_{92}$ & $V_{71}$ & $V_{84}$ & $V_{45}$ & $I_{31-32}$ & $I_{32-71}$ & $I_{33-34}$ & $I_{45-46}$ & $I_{84-911}$ & $I_{92-75}$ \\
\hline THD & 0.01 & 0.38 & 0.82 & 0.53 & 0.52 & 0.55 & 0.18 & 0.10 & 2.49 & 15.49 & 1.84 & 14.22 \\
\hline
\end{tabular}

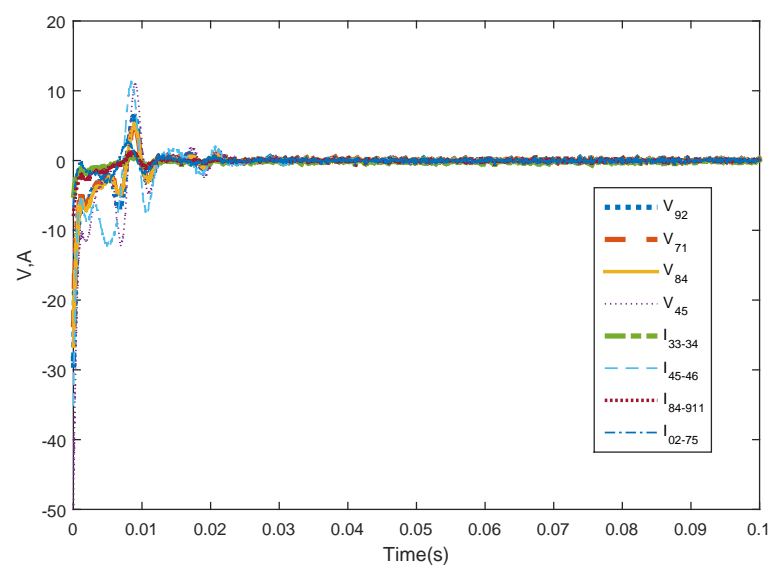

Fig. 7. Estimation errors for noisy measurements

errors approach zero and this validates the accuracy of estimates. The noise is extracted from the harmonic estimates as shown in the estimation error. Thus, this verifies the capability of the proposed iterative observer algorithm to adapt to noisy measurements.

\section{CONCLUSION}

In this paper, a method of simultaneous harmonic estimation of multiple measurements has been proposed. The method utilises the iterative observer, which operates on the principle that an observer has been previously designed for estimation of the process without the consideration of the harmonic disturbance. Simulation results show that the iterative observer is a fast, reliable and effective method for carrying out harmonic estimation in time-varying signals. In addition, this approach accurately estimates the harmonic propagation throughout a power system by ensuring that the system measurements are placed to ensure complete observability. Various scenarios which may be applicable to actual power systems were simulated to verify the accuracy and robustness of the iterative observer algorithm. This algorithm may also be applied to estimation of periodic disturbances in transmission and subtransmission systems and general cases of periodic disturbance estimation in multiple outputs. 


\section{REFERENCES}

Abu-Hashim, R., Burch, R., Chang, G., Grady, M., Gunther, E., Halpin, M., Harziadonin, C., Liu, Y., Marz, M., Ortmeyer, T., Rajagopalan, V., Ranade, S., Ribeiro, P., Sim, T., and Xu, W. (1999). Test Systems For Harmonic Modelling And Simulation. IEEE Transactions on Power Delivery, 14(2):579-587.

Arrillaga, J., Watson, N. R., and Chen, S. (2001). Power System Quality Assessment. Wiley, Chichester, England.

Castillo, E., Conejo, A., Pruneda, R., and Solares, C. (2006). Observability Analysis in State Estimation: A Unified Numerical Approach. IEEE Transactions on Power Systems, 21(2):877-886.

Chang, G., Chen, C., and Liang, Q. (2009). A two-stage ADALINE for harmonics and interharmonics measurement. IEEE Transactions on Industrial Electronics, 56(6):22202228.

Chang, G., Chen, C., Liu, Y., and Wu, M. (2008). Measuring power system harmonics and interharmonics by an improved fast fourier transform-based algorithm. IET Generation, Transmission \& Distribution, 2(2):193-201.

Chang, Y., Hsieh, Y., and Moo, C. (2000). Truncation effects of FFT on estimation of dynamic harmonics in power system. In . International Conference on Power System Technology Proceedings. PowerCon, volume 3, pages 1155-1160.

Chen, B., Pin, G., and Parisini, T. (2014). An adaptive observer-based estimator for multisinusoidal signals. In Proceedings of the American Control Conference, pages 3450-3455.

Chen, C. I. and Chen, Y. C. (2014). Comparative study of harmonic and interharmonic estimation methods for stationary and time-varying signals. IEEE Transactions on Industrial Electronics, 61(1):397-404.

Cisneros-Magaña, R. and Medina, A. (2012). Time-domain harmonic state estimation based on the Kalman filter Poincaré map and extrapolation to the limit cycle. IET Generation, Transmission \& Distribution, 6(12):1209-1217.

Ding, Z. (2008). Asymptotic rejection of finite frequency modes of general periodic disturbances in output feedback nonlinear systems. Automatica, 44(9):2317-2325.

Friedland, B. (1987). Control System Design: An Introduction to State Space Methods. 


\section{McGraw Hill.}

Gou, B. and Abur, A. (2001). An improved measurement placement algorithm for network observability. IEEE Transactions on Power Systems, 16(4):819-824.

Harris, F. (1978). On the use of windows for harmonic analysis with the discrete fourier transform. Proceedings of the IEEE, 66(1):51-83.

IEEE (1993). IEEE Recommended Practices and Requirements for Harmonic Control in Electrical Power Systems. IEEE Std 519-1992, pages 1-112.

Jain, S., Saxena, D., and Singh, S. (2011). Adaptive wavelet neural network based harmonic estimation of single-phase systems. IEEE transactions on Power and Energy Systems, pages $1-7$.

Jain, S., Singh, S., and Singh, J. (2013). An Adaptive Time-Efficient Technique for Harmonic Estimation of Nonstationary Signals. IEEE Transactions on Industrial Electronics, 60(8):3295-3303.

Jain, S. K. and Singh, S. N. (2013). Fast Harmonic Estimation of Stationary and Time-Varying Signals Using EA-AWNN. IEEE Transactions on Instrumentation and Measurement, 62(2):335-343.

Kanieski, J., Cardoso, R., Pinheiro, H., and Grundling, H. (2013). Kalman filter-based control system for power quality conditioning devices. IEEE Transactions on Industrial Electronics, 60(11):5214-5227.

Luenberger, D. (1966). Observers for multivariable systems. IEEE Transactions on Automatic Control, 11(2):190-197.

Ma, H. and Girgis, A. (1996). Identification and tracking of harmonic sources in a power system using a Kalman filter. IEEE Transactions on Power Delivery, 11(3):1659-1665.

Mamis, M. (2003). State-space transient analysis of single-phase transmission lines with corona. In Proceedings of International Conference on Power Systems Transients.

PowerWorld (2012). version 16. Powerworld Corporation, 2001 South first street, Champaign, Illinois, USA.

Qian, H., Zhao, R., and Chen, T. (2007). Interharmonics analysis based on interpolating windowed FFT algorithm. IEEE Transactions on Power Delivery, 22(2):1064-1069.

Ray, P. and Subudhi, B. (2012). Ensemble-Kalman-Filter-Based Power System Harmonic 
Estimation. IEEE Transactions on Instrumentation and Measurement, 61(12):3216-3224.

Regulski, P. and Terzija, V. (2012). Estimation of frequency and fundamental power components using an unscented Kalman filter. IEEE Transactions on Instrumentation and Measurement, 61(4):952-962.

Reza, M., Ciobotaru, M., and Agelidis, V. (2012). Frequency adaptive linear Kalman filter for fast and accurate estimation of grid voltage parameters. In 2012 IEEE International Conference on Power System Technology (POWERCON), pages 1-6.

Selvajyothi, K. and Janakiraman, P. A. (2008). Extraction of Harmonics Using Composite Observers. IEEE Transactions on Power Delivery, 23(1):31-40.

Shafiee Rad, M., Mokhtari, H., and Karimi, H. (2013). A new algorithm for optimal measurement placement, observability analysis and Harmonic State Estimation in power systems. 4th Annual International Power Electronics, Drive Systems and Technologies Conference, pages 518-523.

Sodhi, R., Srivastava, S., and Singh, S. (2010). Optimal PMU placement method for complete topological and numerical observability of power system. Electric Power Systems Research, 80:1154-1159.

Ujile, A. and Ding, Z. (2014). An iterative observer for harmonic estimation in power distribution networks. In IEEE PES Transmission and distribution conference and exposition, Chicago, USA. IEEE.

Wen, X., Guo, L., and Zhang, Y. (2011). Estimation of unknown sinusoidal disturbances using two-step nonlinear observer. In Proceedings of the 30th Chinese Control Conference, CCC 2011, pages 6181-6186.

Wu, Z., Liu, M., and Amara, F. B. (2014). Youla parameterized adaptive regulation against unknown multiple narrow-band disturbances. Transactions of the Institute of Measurement and Control, 36(2):206-215. 